\title{
Implicit finite difference approximation for time Fractional heat conduction under boundary Condition of second kind
}

\author{
T.N. Mishra ${ }^{1 *}$, K.N. Rai ${ }^{2}$ \\ ${ }^{1}$ DST-CIMS, Faculty of Science, BHU, Varanasi \\ ${ }^{2}$ Mathematical Sciences, IIT BHU, Varanasi \\ *Corresponding author E-mail: t.mishra01@gmail.com
}

Copyright (C)2015 Mishra and Rai. This is an open access article distributed under the Creative Commons Attribution License, which permits unrestricted use, distribution, and reproduction in any medium, provided the original work is properly cited.

\begin{abstract}
The time fractional heat conduction in an infinite plate of finite thickness, when both faces are subjected to boundary conditions of second kind, has been studied. The time fractional heat conduction equation is used, when attempting to describe transport process with long memory, where the rate of heat conduction is inconsistent with the classical Brownian motion. The stability and convergence of this numerical scheme has been discussed and observed that the solution is unconditionally stable. The whole analysis is presented in dimensionless form. A numerical example of particular interest has been studied and discussed in details.
\end{abstract}

Keywords: Boundary condition of second kind; Caputo fractional derivative; Implicit finite difference scheme; Time fractional heat conduction; Unconditionally stable.

\section{Introduction}

The fractional calculus is a name for the theory of integral and derivatives of arbitrary orders, which unify and generalize the notions of integer-order differentiation and n-fold integration. In recent years, fractional calculus has been taken by Scientist and Engineers and applied in an increasing numbers of fields, namely in the field of material and mechanics, signal processing, anomalous diffusion, biological system, finance, hydrology and many others see $[18],[12],[24],[27],[21],[11],[1]$.

Fractional sub-diffusion equation is a class of anomalous diffusive system, which is obtained from the classical heat conduction equation by replacing the first orders time derivative to a fractional derivative of order $\alpha$ with $0<\alpha<1$ (in Riemann-Liouville or Caputo sense). It is a known fact that the anomalous diffusion is characterized by a diffusion constant and the mean square displacement of diffusing species in the form

$\left\langle x^{2}(t)\right\rangle \sim t^{\alpha}, \quad t \rightarrow \infty$

where, $\alpha(0<\alpha<1)$ is the anomalous diffusion exponent.

Numerical approaches to different types of fractional diffusion models have been increasingly appearing in literature. Meerchaert [13], considered the stochastic solution of space-time fractional diffusion equation. Huang and Liu [6], considered the time fractional diffusion equation in whole space and also in half space. Yuste and Acedo [26], and Yuste [25], presented an explicit scheme and weighted average finite difference methods for the 
fractional diffusion equation and analyzed these two schemes stability by von Neumann method. Zhuang and Liu [30], approximated the time fractional diffusion equation by implicit difference method. For the fractional sub-diffusion equation Chen and Liu [3], constructed the difference scheme based on Grunwald-Letnikov formula and showed the stability and convergence of the difference scheme using the Fourier method. Zhuang et al. [31], introduced a new way for solving sub-diffusion equation by integration of the original equation on the both sides to obtain an implicit numerical method. Stability and convergence of the scheme were proved by the energy method. Murio [14] constructed implicit finite difference approximation for time fractional diffusion equations but in showing stability of the method by Fourier method he made some flaw. Ding and Zhang [4] showed the stability of implicit finite difference approximation for time fractional diffusion equations. Chen et al. [2] constructed finite difference method for the fractional reaction-sub-diffusion equation. Zhang [28] considered the unconditionally stable finite difference method for fractional partial differential equation. Singh et al. [23] solved the bioheat equations by finite difference method and homotopy perturbation method.

The work mentioned above are dealing with Dirichlet boundary conditions, where no boundary discretization errors are involved. However, for Neumann boundary condition (i.e boundary condition of second kind), the discretization of boundary conditions must dealt with carefully to match global accuracy. Langlands and Henry [9] developed an implicit difference scheme with convergence order $o\left(\tau+h^{2}\right)$ based on $L_{1}$ approximation for RiemannLiouville fractional derivative and numerically verified the unconditional stability of difference scheme but without global convergence analysis. Recently, Zhao and Sun [29] proposed a Box-type scheme for solving a class of fractional sub-diffusion equation with homogeneous Neumann boundary conditions. Since many application problems in science and engineering involve Neumann boundary conditions [9] [20],[15], such as zero flow or specified flow flux condition. Thus, it is very desirable to use high-order algorithms for efficient computations of the numerical solution of this kind of problem. This motivates us to consider the implicit finite difference approximation for spatial discretization.

The main purpose of this paper is to construct a mathematical model for time fractional heat conduction in an infinite plate and discuss the anomalous diffusion, sub-diffusion and also discuss the effect of limiting value of time fractional constant $\alpha$ by taking a particular example. The rest of the paper is organized as follows. Section 1 deals fundamental of fractional calculus, time fractional heat conduction equation and a mathematical model of a boundary value problem governing the process of time fractional heat conduction in an infinite plate of finite thickness whose both faces are subjected to boundary conditions of second kind. An implicit finite difference scheme is given in section 2. Stability and convergence of the scheme is given in section 3. Section 4 contains numerical computation and discussion. The conclusion and future research plan is given in section 5 .

\subsection{Fundamental of fractional calculus}

The Grunwald Letnikov fractional derivative [18] ${ }_{a} D_{t}^{\alpha} g(t)$, of order $\alpha>0$, of a function $g(t)$ is defined as

${ }_{a} D_{t}^{\alpha} g(t)=\sum_{k=0}^{m} \frac{g^{k}(a)(t-a)^{(-\alpha+k)}}{\Gamma(-\alpha+k+1)}+\frac{1}{\Gamma(-\alpha+k+1)} \int_{a}^{t}(t-\tau)^{m-\alpha} g^{m+1}(\tau) d \tau$

This definition of fractional derivative is defined under the assumption that functions are $m+1$ - times continuously differentiable in the closed interval [ $\mathrm{a}, \mathrm{t}$ ] where, $m$ is an integer number satisfying the condition $m>\alpha-1$. But such type of functions are very narrow.

Let $[\mathrm{a}, \mathrm{t}]$ be a finite interval on the real axis $\Re$. The Riemann-Liouville (R-L) fractional derivative $[18]{ }_{a}^{R L} D_{t}^{\alpha} g(t)$, of order $\alpha>0$, of a function $g(t)$ is defined by

${ }_{a}^{R L} D_{t}^{\alpha} g(t)=\frac{d^{n}}{d t^{n}}\left[\frac{1}{\Gamma(n-\alpha)}\right] \int_{a}^{t}(t-\tau)^{n-\alpha-1} g(\tau) d \tau, \quad(n-1<\alpha \leq n)$

The Riemann-Liouville approach have played an important role in the development of theory of fractional calculus due to their applications in pure mathematics. However, R-L approach lead to initial conditions containing the limit values at the lower terminal $t=a$ as

$\lim _{t \rightarrow a}{ }_{a}^{R L} D_{t}^{\alpha-j} g(t)=\overline{c_{j}}, \quad j=1,2, \ldots n$.

where, $\overline{c_{j}}$ are given constants. The above initial value problems with such initial conditions can be successfully solved mathematically but their solutions have no known physical interpretation due to which they are useless. 
The Caputo fractional derivative [18] ${ }_{a}^{C} D_{t}^{\alpha} g(t)$, of order $\alpha$, of a function $g(t)$ is defined as

${ }_{a}^{C} D_{t}^{\alpha} g(t)=\frac{1}{\Gamma(\alpha-n)} \int_{a}^{t}(t-\tau)^{n-\alpha-1} g^{n}(\tau) d \tau, \quad n-1<\alpha \leq n$

Under natural conditions on the function $g(t)$, for $\alpha \rightarrow n$ the Caputo derivative becomes a conventional n-th derivative of the function $g(t)$. The main advantage of Caputo's approach is that the initial conditions for fractional differential equations with Caputo derivatives takes on the same form as for integer-order differential equations, i.e. contain the limit values of integer-order derivatives of unknown functions at the lower terminal $t=a$, due to this in present study we take Caputo fractional derivative.

\subsection{Time fractional heat conduction equation}

The classical theory of heat conduction is based on Fourier law, $\vec{q}=-\nabla T$, relating to the heat flux vector $(\vec{q})$ to the temperature gradient $(\nabla T)$, where, thermal conductivity $\mathrm{K}$ is a geometry-independent coefficient and mainly depends on the composition and structure of the material and the temperature. In many one dimensional systems with total momentum conservation, the heat conduction equation does not obey the Fourier law and heat conductivity depends on the system size [8]. Such size dependent thermal conductivity has been observed in theoretical models, such as the harmonic chains [22], the FPU- $\beta$ model [10] and the hard point gas model [5].

The heat conduction equation is a combination of conservation equation of energy and conduction law. The energy conservation is expressed locally by

$-\nabla \vec{q}(t)=\rho c_{b} \frac{\partial T}{\partial t}$

where, $c_{b}$, is the specific heat capacity at constant pressure and $\rho$, is the density. The theory of heat conduction proposed by Norwood [16], the corresponding generalization of Fourier law,

$\vec{q}(t)=-K \int_{0}^{t} a(t-\tau) \nabla T(\tau) d \tau$

where, $a(t-\tau)$ is the kernel. The time non local dependence between the heat flux vector and the temperature gradient with long tale power kernel [19],

$\vec{q}(t)=\frac{-K}{\Gamma(\alpha)} \int_{0}^{t}(t-\tau)^{\alpha-1} \frac{\partial \nabla T(\tau)}{\partial t} d \tau, \quad 0<\alpha \leq 1$

Considering the definition of Caputo fractional derivative for $0<\alpha \leq 1$

${ }_{0}^{c} D_{t}^{\alpha} g(t)=\frac{1}{\Gamma(1-\alpha)} \int_{0}^{t} \frac{g^{1}(\tau)}{(t-\tau)^{\alpha}} d \tau, \quad 0<\alpha \leq 1$

Now equation (6) becomes,

$\vec{q}(t)=-K\left({ }_{0}^{c} D_{t}^{\alpha-1} \nabla T\right)$

In uni-dimensional case, the gradient of temperature in orthogonal curvilinear coordinate system is given by [7]

$\nabla T=x \frac{1}{h_{1}} \frac{\partial T}{\partial x}$

here,the coefficients $h_{1}$ is called the scale factors and can be evaluated by,

$h_{1}^{2}=1$ 
The heat flux vector $\vec{q}$ becomes,

$\vec{q}=-K\left({ }_{0}^{c} D_{t}^{1-\alpha} x \frac{1}{h_{1}} \frac{\partial T}{\partial x}\right)$

Therefore divergence of heat flux vector $\vec{q}$ in orthogonal curvilinear coordinate system is given by

$$
\nabla \vec{q}=-\frac{1}{h_{1}}\left[\frac{\partial}{\partial x} K\left({ }_{0}^{c} D_{t}^{1-\alpha} \frac{1}{h_{1}} \frac{\partial T}{\partial x}\right)\right.
$$

Then, the fractional heat conduction equation in orthogonal curvilinear coordinate system is given by substituting the result of Eq. (11) into Eq. (4) i.e.

$$
\frac{1}{h_{1}}\left[\frac{\partial}{\partial x} K\left({ }_{0}^{c} D_{t}^{1-\alpha} \frac{1}{h_{1}} \frac{\partial T}{\partial x}\right)\right]=\rho c_{b} \frac{\partial T}{\partial t}
$$

\subsection{Mathematical model of heat conduction in an infinite plate of finite thickness}

The transport process in an infinite plate of finite thickness $2 R$, with the long memory where, the rate of heat conduction in the body is inconsistent with the classical Brownian motion model, is considered. At initial instant, the temperature of the body is a function of space coordinate and the faces are subjected to boundary condition of second kind. The mathematical model describing this anomalous heat conduction in presence of internal heat source, in dimensionless form can be written as:

$$
\begin{aligned}
& \frac{\partial^{\alpha} \theta}{\partial F_{0}^{\alpha}}=\frac{\partial^{2} \theta}{\partial x^{2}}+P_{0}\left(x, F_{0}\right) \\
& (-1)^{j} \frac{\partial \theta}{\partial x}=k_{i_{j}}\left(F_{0}\right), \quad x=(-1)^{j}, \quad j=1,2 \\
& \theta(x, 0)=f(x), \quad F_{0}>0
\end{aligned}
$$

\section{Nomenclature, dimensional variable and similarity criteria}

$\begin{array}{ll}a_{q} & \text { thermal diffusivity }\left(\mathrm{m}^{2} \mathrm{~s}^{-1}\right) \\ b_{1} & \text { time dependent coefficient }\left(s^{-1} K\right) \\ c_{b} & \text { specific heat capacity }(\mathrm{J} / \mathrm{kg} K) \\ { }_{a} D_{t}^{\alpha} & \text { Grunwald Letnikove fractional derivative } \\ { }_{a} D_{t}^{\alpha} & \text { Riemann-Liouville fractional derivative } \\ { }_{a}^{C} D_{t}^{\alpha} & \text { Caputo fractional derivative } \\ \mathrm{g}(\mathrm{t}) & \text { a continuous differentiable function of } t \\ h_{1} & \text { scale factor } \\ K & \text { thermal conductivity }(\mathrm{W} / \mathrm{m} \mathrm{K}) \\ k & \text { temporal grid size } \\ Q & \text { heat source }(J) \\ T & \text { temperature }(K) \\ T_{c} & \text { ambient temperature }(K) \\ \Delta x & \text { spatial grid size }(=h) \\ \rho & \text { density }\left(\mathrm{kg} m^{-3}\right)\end{array}$

$\begin{array}{ll}f(x) & \text { dimensionless initial temperature } \\ F_{0} & \text { Fourier number, } \frac{a_{q} t}{R^{2}} \\ k_{i}\left(F_{0}\right) & \text { Kirpichev number, } \frac{q(\tau) R}{K\left(T_{c}-T_{0}\right)} \\ k_{i_{1}}\left(F_{0}\right) & \text { dimensionless heat flux at boundary } \mathrm{x}=-1 \\ k_{i_{2}}\left(F_{0}\right) & \text { dimensionless heat flux at boundary } \mathrm{x}=1 \\ P_{0} & \text { Pomerantsev number, } \frac{Q(x, \tau) R^{2}}{K\left(T_{c}-T_{0}\right)} \\ P_{d} & \text { Predvoditelev number, } \frac{b_{1} R^{2}}{a_{q} \Delta T} \\ x & \text { dimensionless spatial coordinate, }, \frac{r}{R} \\ \theta & \text { dimensionless temperature, } \frac{T-T_{0}}{T_{c}-T_{0}} \\ q(t) & \text { heat flux }\left(W / m^{2}\right) \\ 2 \mathrm{R} & \text { thickness of the infinite plate }(m) \\ T_{0} & \text { initial temperature }(K) \\ \nabla T & \text { temperature gradient }(K / \mathrm{m}) \\ \alpha & \text { order of fractional derivative in }(\mathrm{n}-1, \mathrm{n}] \\ a, t & \text { lower and upper terminals of fractional order }\end{array}$

\section{Implicit finite difference scheme}

To establish the numerical approximation scheme, let $\Delta x=h=\frac{2}{X}>0$ and $k=\frac{1}{M}$ be the grid size in space and time direction respectively. The grid points in the space interval $[-1,1]$ are the numbers $x_{i}=i h, i=1,2,3 \ldots p$ and grid points in the time interval $\left[0, F_{0}\right]$ are labelled $F_{0_{n}}=n k, n=1,2, . ., m$. The values of the functions $\theta$ and $f$ 
at grid points are denoted by $\theta_{i}^{n}=\theta\left(x_{i}, F_{0_{n}}\right)$ and $f_{i}=f\left(x_{i}\right)$, respectively. In the differential Eq. (12), we have adopted a symmetric second difference quotient in space at level $F_{0}=F_{0_{n+1}}$ for approximating the second order space derivative. The time fractional derivative term can be approximated by the following scheme:

$$
\begin{aligned}
& \frac{\partial^{\alpha} \theta\left(x_{i}, F_{0_{n+1}}\right)}{\partial F_{0}^{\alpha}}=\frac{1}{\Gamma(1-\alpha)} \int_{0}^{F_{0_{n+1}}} \frac{\partial \theta\left(x_{i}, s\right)}{\partial F_{0}}\left(F_{0_{n+1}}-s\right)^{-\alpha} d s \\
& =\frac{1}{\Gamma(1-\alpha)} \sum_{j=0}^{n} \int_{j k}^{(j+1) k}\left[\frac{\theta_{i}^{j+1}-\theta_{i}^{j}}{k}\right]\{(n+1) k-s\}^{-\alpha} d s \\
& =\frac{1}{\Gamma(1-\alpha)} \frac{1}{(1-\alpha)} \sum_{j=0}^{n}\left(\left[\frac{\theta_{i}^{j+1}-\theta_{i}^{j}}{k}\right]\left[(n-j+1)^{1-\alpha}-(n-j)^{1-\alpha}\right]\right) k^{1-\alpha} \\
& =\frac{1}{\Gamma(1-\alpha)} \frac{1}{(1-\alpha)} \frac{1}{k^{\alpha}}\left(\theta_{i}^{j+1}-\theta_{i}^{j}\right)+\frac{1}{\Gamma(1-\alpha)} \frac{1}{(1-\alpha)} \frac{1}{k^{\alpha}} \sum_{j=1}^{n}\left(\theta_{i}^{j+1}-\theta_{i}^{j}\right)\left[(n-j+1)^{1-\alpha}-(n-j)^{1-\alpha}\right]
\end{aligned}
$$

By taking $\sigma_{\alpha, k}=\frac{1}{\Gamma(1-\alpha)} \frac{1}{(1-\alpha)} \frac{1}{k^{\alpha}}$ and shifting indices $w_{j}^{\alpha}=(j)^{1-\alpha}-(j-1)^{1-\alpha}$, and define

$$
L_{h, k}^{\alpha} \theta\left(x_{i, F_{0_{n+1}}}\right)=\sigma_{\alpha, k} \sum_{j=0}^{n} w_{j}^{\alpha}\left(\theta_{i}^{n+1-j}-\theta_{i}^{n-j}\right)
$$

We have,

$$
\begin{aligned}
& \left|\frac{\partial^{\alpha} \theta\left(x_{i}, F_{0_{n+1}}\right)}{\partial F_{0}^{\alpha}}-L_{h, k}^{\alpha} \theta\left(x_{i, F_{0_{n+1}}}\right)\right| \\
& \leq \frac{1}{(1-\alpha)} \sum_{j=0}^{n} \int_{F_{0_{j}}}^{F_{0_{j+1}}}\left|\frac{\partial \theta\left(x_{i}, \xi\right)}{\partial \xi}-\frac{\theta\left(x_{i}, F_{0_{j+1}}\right)-\theta\left(x_{i}, F_{0_{j}}\right)}{k}\right| \frac{d \xi}{\left(F_{0_{n+1}}-\xi\right)^{\alpha}} \\
& \leq \frac{C_{1}}{\Gamma(1-\alpha)} k \sum_{j=0}^{n} \int_{F_{0_{j}}}^{F_{0_{j+1}}} \frac{d \xi}{\left(F_{0_{n+1}}-\xi\right)^{\alpha}} \\
& \leq \frac{C_{1}}{\Gamma(1-\alpha)} k \int_{0}^{F_{0_{j+1}}} \frac{d \xi}{\left(F_{0_{n+1}}-\xi\right)^{\alpha}} \\
& \left|\frac{\partial^{\alpha} \theta\left(x_{i}, F_{0_{n+1}}\right)}{\partial F_{0}^{\alpha}}-L_{h, k}^{\alpha} \theta\left(x_{i, F_{0_{n+1}}}\right)\right| \leq \bar{C}_{1} k
\end{aligned}
$$

where, $C_{1}$ and $\bar{C}_{1}$ are constants.

On using finite central difference formula and approximating derivatives at $x_{0}$ and $x_{n}$, the differential Eqs. (12)-(14), reduces in the vector-matrix form as follows:

$A \Theta^{1}=\bar{\Theta}^{0}+P^{1}$ 
$A \Theta^{n+1}=c_{1} \bar{\Theta}^{n}+c_{2} \bar{\Theta}^{n-1}+\ldots \ldots \ldots . . .+c_{n} \bar{\Theta}^{1}+d_{n+1} \bar{\Theta}^{0}+P^{n}, \quad n \geq 1$

$\Theta^{0}=f$

where,

$\bar{\Theta}^{n}=\Theta^{n}\left(\sigma_{\alpha, k}\right)_{1 \times 1}$

$\Theta^{n}=\left(\begin{array}{llllllll}\theta_{1}^{n} & \theta_{2}^{n} & \theta_{3}^{n} & \cdot & \cdot & \cdot & \theta_{p-2}^{n} & \theta_{p-1}^{n}\end{array}\right)^{t}$

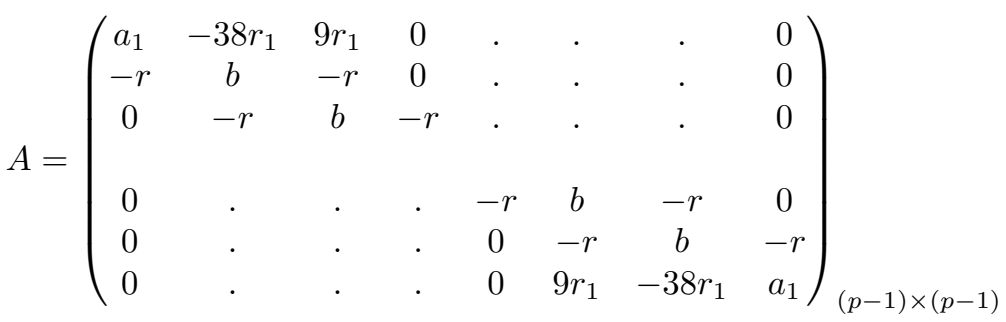

$P^{n}=\left(\begin{array}{llllllllll}P_{1}^{n}+20 h k_{i, 1} r_{1} & P_{2}^{n} & P_{3}^{n} & \cdot & . & . & P_{p-3}^{n} & P_{p-2}^{n} & P_{p-1}^{n}+20 h k_{i, 2} r_{1}\end{array}\right)^{t}$

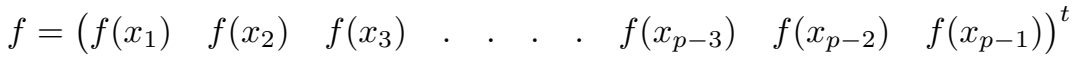

$c_{j}=\left(w_{j}^{\alpha}-w_{j+1}^{\alpha}\right), d_{n}=w_{n}^{\alpha}, a_{1}=\left(\sigma_{\alpha, k}+29 r_{1}\right), b=\left(\sigma_{\alpha, k}+2 r\right), r_{1}=\frac{1}{21 h^{2}}, r=\frac{1}{h^{2}}$ and $X^{t}$ be the transpose of matrix $X$.

From Eqs. (16)-(18) we observe that at each time step, we get a triangular system of linear equations which utilize all the history of the computed solution up to that time. In present analysis, Mathematical software MATLAB 7.0 has been used to obtain dimensionless temperature $\Theta^{n}$.

\section{Stability and convergence}

In this section we will analyze the stability and convergence of the implicit finite difference scheme for time fractional heat conduction equation. From Eqs. (16)-(18), and by using the property of the function $g(x)=x^{1-\alpha},(1 \leq x)$ and

$1=w_{1}^{\alpha}>w_{2}^{\alpha}>w_{3}^{\alpha}>w_{4}^{\alpha}>\ldots . . \longrightarrow 0$

we have the following,

$\sum_{j=1}^{n} c_{j}=1-w_{n+1}^{\alpha}$

$\sum_{j=1}^{\infty} c_{j}=1,1-2^{1-\alpha}>2^{2-\alpha}-3^{1-\alpha}-1=c_{1}>c_{2}>c_{3} \ldots \longrightarrow 0$

- Matrix A is strictly diagonally dominant with positive diagonal term and non positive off-diagonal terms. Hence the Eqs. (16)-(18) can be solved.

- The solution $\theta_{i}^{n}(i=1,2,3 \ldots p-1 ; n=1,2,3 \ldots m)$ possess non negativity, if $\theta_{0}^{n}$ is non negative.

- Solution $\theta_{i}^{j}$ is conservative, i.e $\sum_{i=-\infty}^{\infty}\left|\theta_{i}^{0}\right|<\infty \Longrightarrow \sum_{i=-\infty}^{\infty}\left|\theta_{i}^{j}\right|=\sum_{i=-\infty}^{\infty}\left|\theta_{i}^{0}\right|, j \in \aleph$ 
Now we suppose $\tilde{\theta_{i}^{j}}$ is the approximate solution of Eqs. (16)-(18), then the error $\epsilon_{i}^{j}=\tilde{\theta_{i}^{j}}-\theta_{i}^{j}$ satisfies,

$A E^{1}=\bar{E}^{0}$

$A E^{n+1}=c_{1} \bar{E}^{n}+c_{2} \bar{E}^{n-1}+c_{3} \bar{E}^{n-2}+\ldots \ldots \ldots \ldots+c_{n} \bar{E}^{1}+d_{n+1} \bar{E}^{0}, n \geq 1$

where, $\bar{E}^{n}=E^{n}\left(\sigma_{\alpha, k}\right)_{1 \times 1}$

and $E^{n}=\left(\begin{array}{lllllllll}\epsilon_{1}^{n} & \epsilon_{2}^{n} & \epsilon_{3}^{n} & \text {. } & \text {. } & . & \epsilon_{p-2}^{n} & \epsilon_{p-1}^{n}\end{array}\right)^{t}$.

Hence, by the mathematical induction following result can be proved.

Theorem $3.1\left\|E^{n}\right\|_{\infty} \leq\left\|E^{0}\right\|_{\infty}$

Proof : For $n=1$,

$-r \epsilon_{i-1}^{1}+\left(\sigma_{\alpha, k}+2 r\right) \epsilon_{i}^{1}-r \epsilon_{i+1}^{1}=\sigma_{\alpha, k} \epsilon_{i}^{0}$

Let $\left|\epsilon_{l}^{1}\right|=\underbrace{\max }_{1 \leq i \leq p-1}\left|\epsilon_{i}^{1}\right|$ then we have

$\left|\epsilon_{l}^{1}\right| \leq-r\left|\epsilon_{l-1}^{1}\right|+\left(\sigma_{\alpha, k}+2 r\right)\left|\epsilon_{l}^{1}\right|-r\left|\epsilon_{l+1}^{1}\right|$

$\leq\left|-r \epsilon_{l-1}^{1}+\left(\sigma_{\alpha, k}+2 r\right) \epsilon_{l}^{1}-r \epsilon_{l+1}^{1}\right|$

$=\left|\sigma_{\alpha, k} \epsilon_{l}^{0}\right|$

$\left\|E^{1}\right\|_{\infty} \leq\left\|E^{0}\right\|_{\infty}$

Therefore, $\left\|E^{1}\right\|_{\infty} \leq\left\|E^{0}\right\|_{\infty}$.

Suppose that $\left\|E^{j}\right\|_{\infty} \leq\left\|E^{0}\right\|_{\infty}, \quad j=1,2,3 \ldots . n$. Let $\left|\epsilon_{l}^{n+1}\right|=\underbrace{\max }_{1 \leq i \leq p-1}\left|\epsilon_{i}^{n+1}\right|$, then we also have,

$\left|\epsilon_{l}^{n+1}\right| \leq-r\left|\epsilon_{l-1}^{n+1}\right|+\left(\sigma_{\alpha, k}+2 r\right)\left|\epsilon_{l}^{n+1}\right|-r\left|\epsilon_{l+1}^{n+1}\right|$

$\leq\left|-r \epsilon_{l-1}^{n+1}+\left(\sigma_{\alpha, k}+2 r\right) \epsilon_{l}^{n+1}-r \epsilon_{l+1}^{n+1}\right|$

$=\left|c_{1} \epsilon_{l}^{n}+\sum_{j=1}^{n-1} c_{j+1} \epsilon_{l}^{n-j}+d_{n+1} \epsilon_{l}^{0}\right|$

$\leq c_{1}\left|\epsilon_{l}^{n}\right|+\sum_{j=1}^{n-1} c_{j+1}\left|\epsilon_{l}^{n-j}\right|+d_{n+1}\left|\epsilon_{l}^{0}\right|$

$\left\|E^{n+1}\right\|_{\infty} \leq c_{1}\left\|E^{n}\right\|_{\infty}+\sum_{j=1}^{n-1} c_{j+1}\left\|E^{n-j}\right\|+w_{n+1}^{\alpha}\left\|E^{0}\right\|_{\infty}$

$\leq\left\{c_{1}+\sum_{j=1}^{n-1} c_{j+1}+w_{n+1}^{\alpha}\right\}\left\|E^{0}\right\|_{\infty}$

$=\left\|E^{0}\right\|_{\infty}$

$\left\|E^{n+1}\right\|_{\infty} \leq\left\|E^{0}\right\|_{\infty}$

Hence, the following theorem is obtained.

Theorem 3.2 The time fractional implicit difference approximation defined by (16) and (18) are unconditionally stable. 


\subsection{Convergence}

To discuss the convergence of implicit finite difference approximation for time fractional heat conduction equation under non-homogeneous boundary conditions of second kind, let $\theta\left(x_{i}, F_{0_{n}}\right)$ be the exact solution of the time fractional heat conduction Eqs. (12)-(14) at mesh point $\left(x_{i}, F_{0_{n}}\right)$. Define $e_{i}^{n}=\theta\left(x_{i}, F_{0_{n}}\right)-\theta_{i}^{n}, i=1,2,3 \ldots . . p-1 ; n=$ $1,2,3 \ldots \ldots m$ and $e^{n}=\left(\sigma_{\alpha, k} e_{1}^{n}, \sigma_{\alpha, k} e_{2}^{n}, \sigma_{\alpha, k} e_{3}^{n} \ldots . . \sigma_{\alpha, k} e_{p-1}^{n}\right)^{t}$. Using $e^{0}=0$, and substitution into (16)-(18) leads to,

$-r e_{i-1}^{1}+\left(\sigma_{\alpha, k}+2 r\right) e_{i}^{1}-r e_{i+1}^{1}=R_{i}^{1}$

$-r e_{i-1}^{n+1}+\left(\sigma_{\alpha, k}+2 r\right) e_{i}^{n+1}-r e_{i+1}^{n+1}=c_{1} e_{i}^{n}+\sum_{j=1}^{n-1} c_{j+1} e_{i}^{n-j}+R_{i}^{n+1}$

where,

$$
\begin{gathered}
R_{i}^{n+1}=\sigma_{\alpha, k} \theta\left(x_{i}, F_{0_{n+1}}\right)-\sigma_{\alpha, k} \theta\left(x_{i}, F_{0_{n}}\right)+\sigma_{\alpha, k} \sum_{j=1}^{n-1} w_{j}^{\alpha}\left[\theta\left(x_{i}, F_{0_{n+1-j}}\right)\right. \\
\left.-\theta\left(x_{i}, F_{0_{n-j}}\right)\right]-r\left[\theta\left(x_{i+1}, F_{0_{n+1}}\right)-2 \theta\left(x_{i}, F_{0_{n+1}}\right)+\theta\left(x_{i-1}, F_{0_{n+1}}\right)\right]-P_{i}^{n+1} \\
=\sigma_{\alpha, k} \sum_{j=0}^{n} w_{j}^{\alpha}\left[\theta\left(x_{i}, F_{0_{n+1-j}}\right)-\theta\left(x_{i}, F_{0_{n-j}}\right)\right]-r\left[\theta\left(x_{i+1}, F_{0_{n+1}}\right)-2 \theta\left(x_{i}, F_{0_{n+1}}\right)+\theta\left(x_{i-1}, F_{0_{n+1}}\right)\right]-P_{i}^{n+1}
\end{gathered}
$$

From Eq. (15), we have

$\sigma_{\alpha, k} \sum_{j=0}^{n} w_{j}^{\alpha}\left[\theta\left(x_{i}, F_{0_{n+1-j}}\right)-\theta\left(x_{i}, F_{0_{n-j}}\right)\right]=\frac{\partial^{\alpha} \theta\left(x_{i}, F_{0_{n+1}}\right)}{\partial F_{0_{n+1}}^{\alpha}}+\bar{C}_{1} k$

and

$\frac{\theta\left(x_{i+1}, F_{0_{n+1}}\right)-2 \theta\left(x_{i}, F_{0_{n+1}}\right)+\theta\left(x_{i-1}, F_{0_{n+1}}\right)}{h^{2}}=\frac{\partial^{2} \theta\left(x_{i}, F_{0_{n+1}}\right)}{\partial x^{2}}+C_{2} h^{2}$

Therefore from Eqs. (19), (20) and (21) we get,

$\left|R_{i}^{n+1}\right| \leq C\left(k^{1+\alpha}+k^{\alpha} h^{2}\right), i=1,2,3 \ldots p-1 ; n=1,2,3 \ldots m$.

where, $\mathrm{C}$ is a constant.

Theorem 3.3 $\left\|e^{n}\right\|_{\infty} \leq C\left(w_{n}^{\alpha}\right)^{-1}\left(k^{1+\alpha}+k^{\alpha} h^{2}\right), n=1,2, \ldots . m$ where, $\left\|e^{n}\right\|_{\infty}=\underbrace{\max }_{1 \leq i \leq p-1}\left|e_{i}^{n}\right|$ and $C$ is a constant.

Proof : We will prove the above theorem by mathematical induction method. For $n=1$, let $\left\|e^{1}\right\|_{\infty}=\left|\sigma_{\alpha, k} e_{l}^{1}\right|=$ $\left|\sigma_{\alpha, k}\right|\left|e_{l}^{1}\right|=\underbrace{\max }_{1 \leq i \leq p-1}\left|\sigma_{\alpha, k} e_{i}^{1}\right|=\left|\sigma_{\alpha, k}\right| \underbrace{\max }_{1 \leq i \leq p-1}\left|e_{i}^{1}\right|$ i.e $\left\|e^{1}\right\|_{\infty}=\left|e_{l}^{1}\right|=\underbrace{\max }_{1 \leq i \leq p-1}\left|e_{i}^{1}\right|$, hence we have

$\left|e_{l}^{1}\right| \leq-r\left|e_{l+1}^{1}\right|+\left(\sigma_{\alpha, k}+2 r\right)\left|e_{l}^{1}\right|-r\left|e_{l-1}^{1}\right|$

$\leq\left|-r e_{l+1}^{1}+\left(\sigma_{\alpha, k}+2 r\right) e_{l}^{1}-r e_{l-1}^{1}\right|$

$=\left|R_{i}^{1}\right|$

$\leq C\left(w_{1}^{\alpha}\right)^{-1}\left(k^{1+\alpha}+k^{\alpha} h^{2}\right)$

Suppose that $\left\|e^{j}\right\|_{\infty} \leq C\left(w_{j}^{\alpha}\right)^{-1}\left(k^{1+\alpha}+k^{\alpha} h^{2}\right), \mathrm{j}=1,2, . . \mathrm{n}$ and $\left|\sigma_{\alpha, k} e_{l}^{n+1}\right|=\underbrace{\max }_{1 \leq i \leq p-1}\left|\sigma_{\alpha, k} e_{i}^{1}\right|$ i.e $\left|e_{l}^{n+1}\right|=\underbrace{\max }_{1 \leq i \leq p-1}\left|e_{i}^{1}\right|$.

Here we note that $\left(w_{j}^{\alpha}\right)^{-1} \leq\left(w_{n}^{\alpha}\right)^{-1}, \mathrm{j}=1,2,3 \ldots . .$. . Then we have

$$
\begin{aligned}
& \left|e_{l}^{n+1}\right| \leq-r\left|e_{l+1}^{n+1}\right|+\left(\sigma_{\alpha, k}+2 r\right)\left|e_{l}^{n+1}\right|-r\left|e_{l-1}^{n+1}\right| \\
& \leq \mid-r e_{l+1}^{n+1}+\left(\sigma_{\alpha, k}+2 r\right) e_{l}^{n+1}-r e_{l-1}^{n+1}
\end{aligned}
$$




$$
\begin{aligned}
& =\left|c_{1} e_{l}^{n}+\sum_{j=1}^{n-1} c_{j+1} e_{l}^{n-j}+R_{i}^{n+1}\right| \\
& \leq c_{1}\left|e_{l}^{n}\right|+\sum_{j=1}^{n-1} c_{j+1}\left|e_{l}^{n-j}\right|+C\left(k^{1+\alpha}+k^{\alpha} h^{2}\right) \\
& \leq c_{1}\left\|e^{n}\right\|_{\infty}+\sum_{j=1}^{n-1} c_{j+1}\left\|e^{n-j}\right\|_{\infty}+C\left(k^{1+\alpha}+k^{\alpha} h^{2}\right) \\
& \leq\left[c_{1}+\sum_{j=1}^{n-1} c_{j+1}+w_{n+1}^{\alpha}\right]\left(w_{n+1}^{\alpha}\right)^{-1} C\left(k^{1+\alpha}+k^{\alpha} h^{2}\right) \\
& =\left(w_{n+1}^{\alpha}\right)^{-1} C\left(k^{1+\alpha}+k^{\alpha} h^{2}\right)
\end{aligned}
$$

Since,

$$
\begin{aligned}
& \lim _{n \rightarrow \infty} \frac{\left(w_{n+1}^{\alpha}\right)^{-1}}{(n+1)^{\alpha}}=\lim _{n \rightarrow \infty} \frac{(n+1)^{-\alpha}}{(n+2)^{1-\alpha}-(n+1)^{1-\alpha}} \\
& =\lim _{n \rightarrow \infty} \frac{(n+1)^{-1}}{\left(1+\frac{1}{n+1}\right)^{1-\alpha}-1} \\
& =\lim _{n \rightarrow \infty} \frac{(n+1)^{-1}}{(1-\alpha)(n+1)^{-1}} \\
& =\frac{1}{1-\alpha},
\end{aligned}
$$

hence, there is a constant $\bar{C}$,such that $\left\|e^{n}\right\|_{\infty} \leq \bar{C}(n+1)^{\alpha}\left(k^{1+\alpha}+k^{\alpha} h^{2}\right)$. Since, $n k \leq F_{0}$ is finite, we obtain the following result.

Theorem 3.4 Let $\theta_{i}^{n}$ be the approximate value of $\theta\left(x_{i}, F_{0_{n}}\right)$ computed by use of the difference scheme (16)-(18) then there is a positive constant $C$ such that $\left|\theta_{i}^{n}-\theta\left(x_{i}, F_{0_{n}}\right)\right| \leq C\left(k+h^{2}\right), i=i, 2,3 \ldots p-1, n=1,2,3 \ldots m$.

\section{Numerical computation and discussion}

Consider the heat conduction equation in an infinite plate of finite thickness with the boundary and initial condition:

$\frac{\partial^{\alpha} \theta}{\partial F_{0}^{\alpha}}=\frac{\partial^{2} \theta}{\partial x^{2}}$

$(-1)^{j} \frac{\partial \theta}{\partial x}=k_{i_{j}}\left(F_{0}\right), \quad x=(-1)^{j}, \quad j=1,2$

$\theta(x, 0)=0, \quad F_{0}>0$

where $k_{i_{1}}\left(F_{0}\right)=k_{i_{2}}\left(F_{0}\right)=k_{i_{1}} \exp \left(-P_{d} F_{0}\right)$

In this study the computation has been made and the results are presented in ten Figures. On the Figures presented in this study, only the parameters whose values different from the reference value are indicated. The selected reference values include $h=0.2, k=\frac{1}{64}, k_{i_{1_{0}}}=k_{i_{2_{0}}}=1.0$.

Variation of dimensionless temperature $\theta$ with space coordinate $x$ is given in Fig. 1. The temperature decreases as $x$ increases and attains a minimum value at centre i.e $x=0$ and then increases symmetrically. Further we observe that for $F_{0}=0.375$, temperature decreases as $\alpha$ increases as shown in Fig. 1(a) whereas for $F_{0}=1.50$, temperature increases as $\alpha$ increases as shown in Fig. 1(b).

From Fig. 2, it is evident that, dimensionless temperature increases and attains a maximum value with increase in time fractional order $\alpha$. A close examination of Figs. 2 and 3 reveal that: 


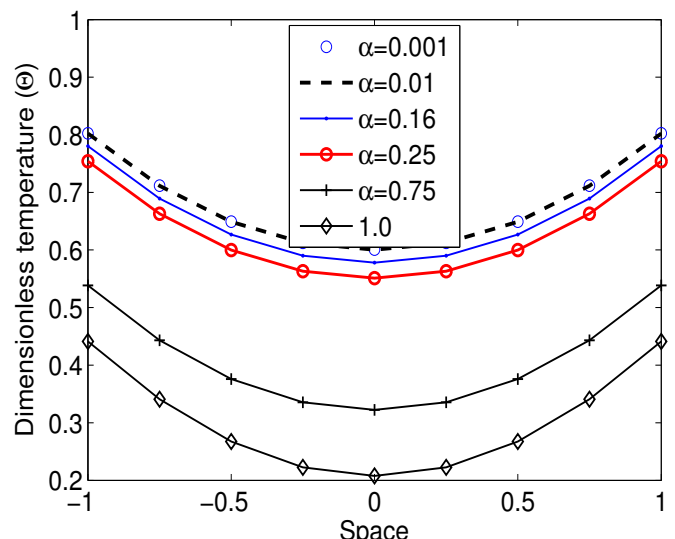

(a) At $P_{d}=1.0, F_{0}=0.375$

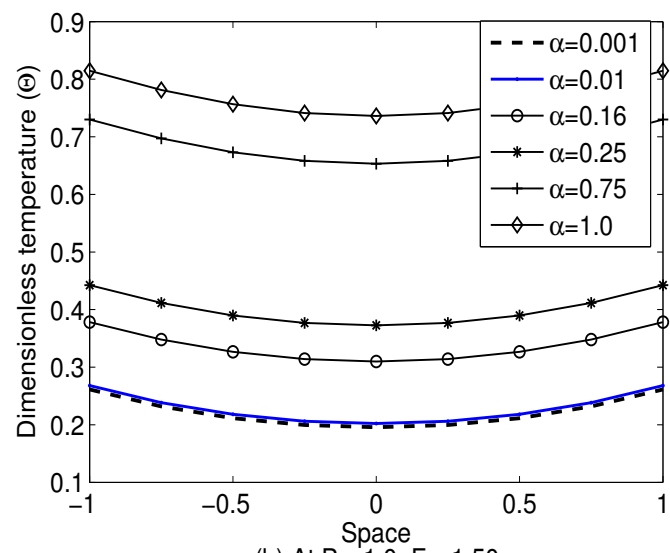

(b) At $P_{d}=1.0, F_{0}=1.50$

Fig. 1: Variation of dimensionless temperature $(\theta)$ with space $(x)$

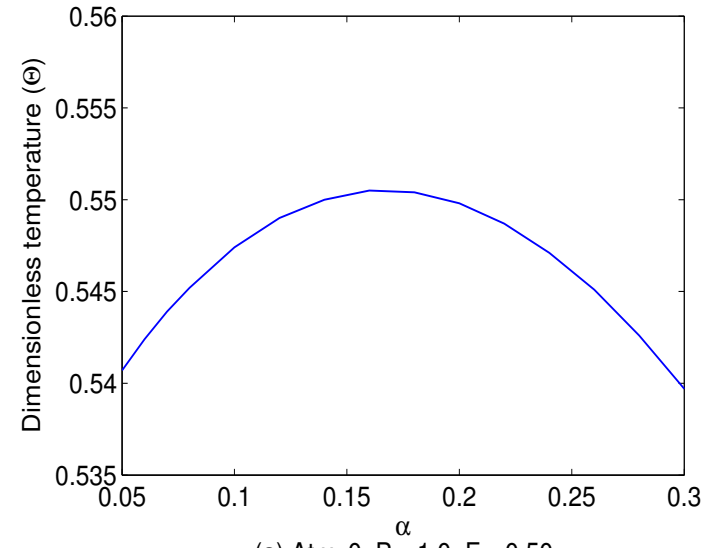

(a) At $x=0, P_{d}^{\alpha}=1.0, F_{0}=0.50$

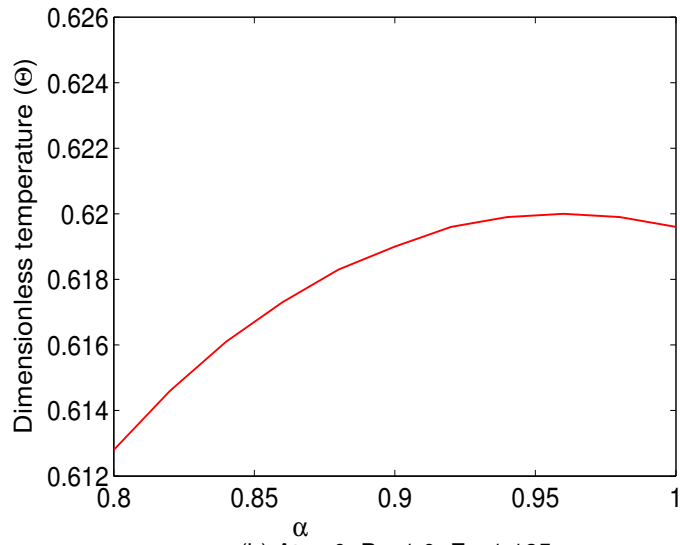

(b) At $x=0, P_{d}=1.0, F_{0}=1.125$

Fig. 2: Variation of dimensionless temperature $(\theta)$ with $\alpha$

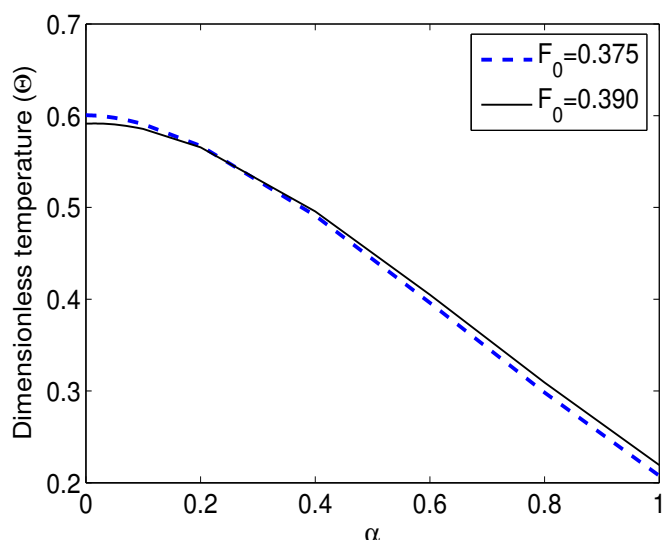

(a) At $\mathrm{x}=0, \mathrm{P}_{\mathrm{d}}=1.0$

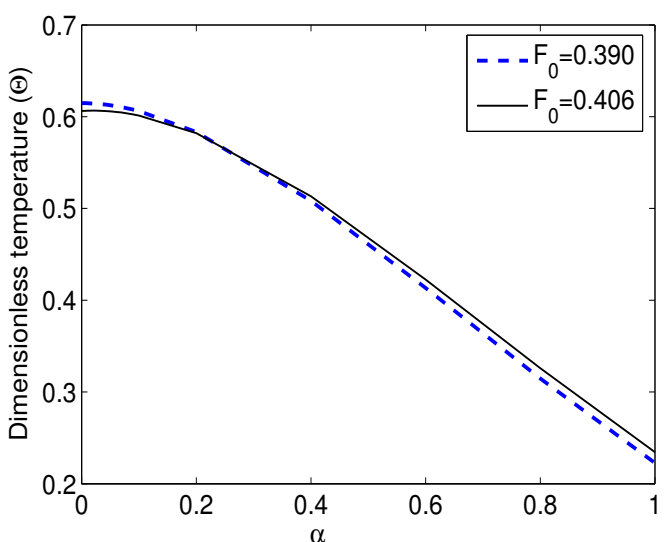

(b) At $x=0, P_{d}=0.9$

Fig. 3: Variation of dimensionless temperature $(\theta)$ with $\alpha$ 


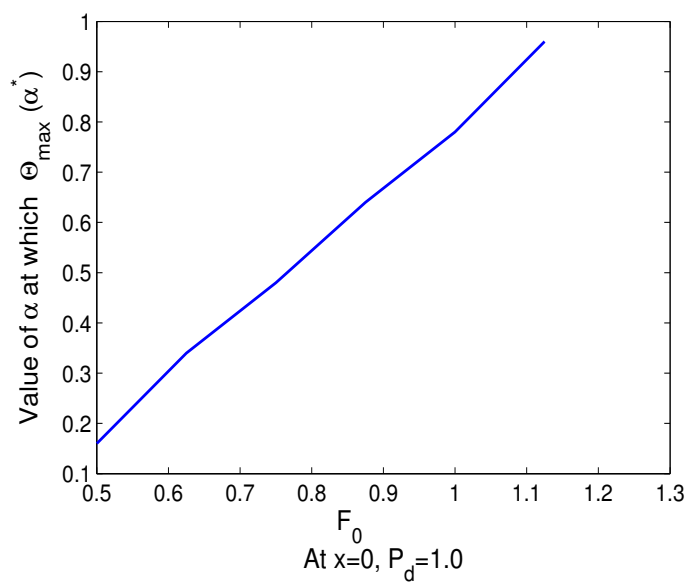

Fig. 4: Variation of $\alpha$ with $F_{0}$ at maximum temperature
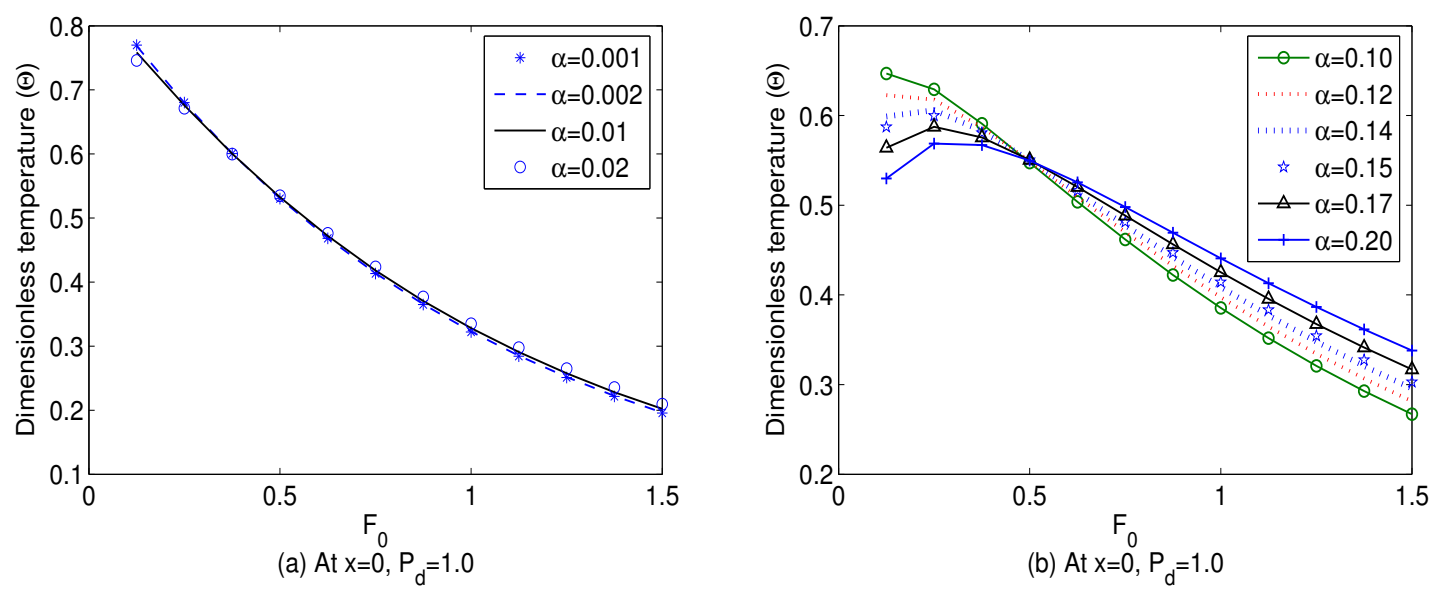

Fig. 5: Variation of dimensionless temperature $(\theta)$ with $F_{0}$

- For $F_{0} \leq 0.375$ (say critical time $F_{0}^{*}$ ) and $P_{d}=1.0$, dimensionless temperature decreases for any small increase in time fractional order $\alpha$ at $x=0$.

- For $F_{0}>0.375$ and $P_{d}=1.0$, dimensionless temperature increases and attains a maximum value for certain time fractional order (say $\alpha^{*}$ ) and decreases further with increase of $\alpha$ as shown in Fig. 3(a).

- When $P_{d}$ decreases, the critical time $F_{0}^{*}$ increases as shown in Fig. 3(b).

- When $F_{0}$ increases from 0.375 , for fixed $P_{d}=1.0$, the value of $\alpha^{*}$ at which maximum temperature attains, increases, as shown in Fig. 4. Also from Fig. 4 we observe that the value of $\alpha$ at which dimensionless temperature is maximum, increases with $F_{0}$, for fixed $P_{d}$.

Variation of dimensionless temperature with $F_{0}$ for different $\alpha$ is given in Fig. 5. For small time fractional order, dimensionless temperature decreases with increase of $F_{0}$ at fixed $P_{d}=1.0$, as shown in Fig. 5(a). A close examination of Fig. 5 reveals that temperature increases and attains a maximum value for certain $F_{0}^{*}$ and then decreases with increase of $\alpha$.

From Fig. 6(a) it is evident that dimensionless temperature increases then decreases gradually with $F_{0}$, whereas, for $\alpha \longrightarrow 1$ i.e. in case of normal diffusion, dimensionless temperature increases with increase in Fourier number $F_{0}$, as shown in Fig. 6(b).

From Fig. 7 , it is evident that dimensionless temperature decreases with increase of $P_{d}$ for any fixed $F_{0}$ and any fixed time fractional order $\alpha \in(0,1]$. At the same time, as $P_{d}$ increases, for small $F_{0}$ and small $\alpha$ very close to zero, dimensionless temperature decreases with increase in $\alpha$ as shown in Fig. 7(a). 


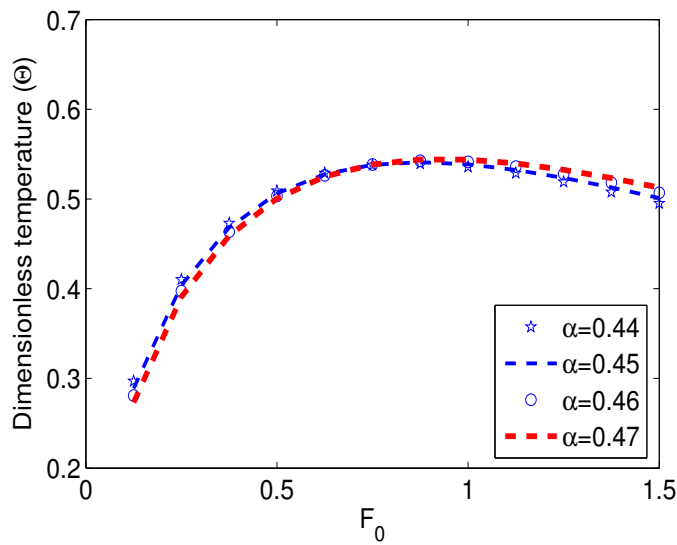

(a) At $\mathrm{x}=0, \mathrm{P}_{\mathrm{d}}=1.0$

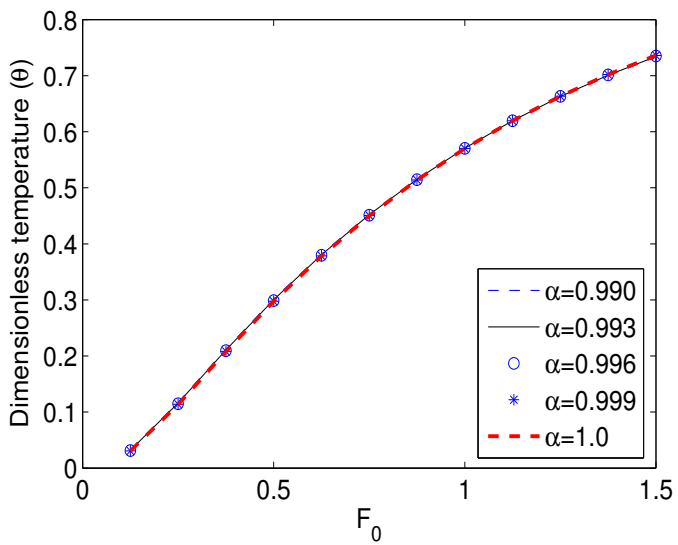

(b) At $x=0, P_{d}=1.0$

Fig. 6: Variation of dimensionless temperature $(\theta)$ with $F_{0}$

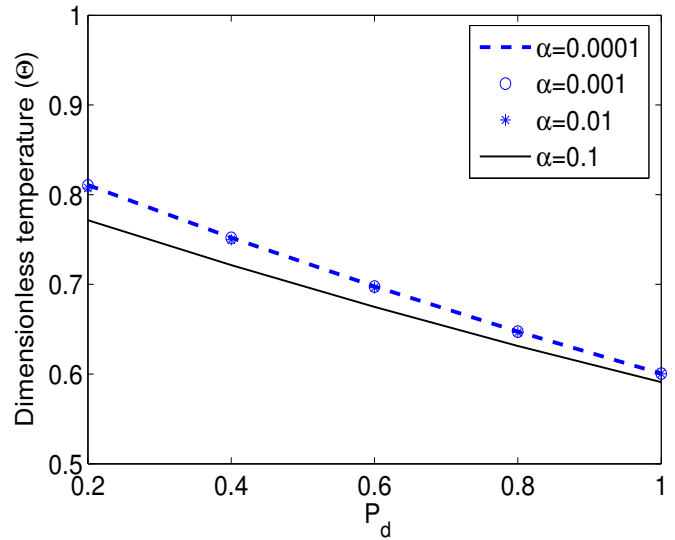

(a) At $\mathrm{x}=0, \mathrm{~F}_{0}=0.375$

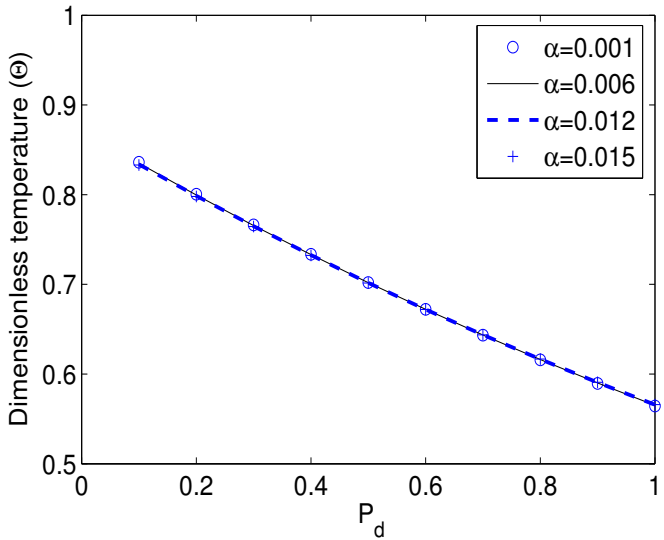

(b) At $\mathrm{x}=0, \mathrm{~F}_{0}=0.437$

Fig. 7: Variation of dimensionless temperature $(\theta)$ with $P_{d}$

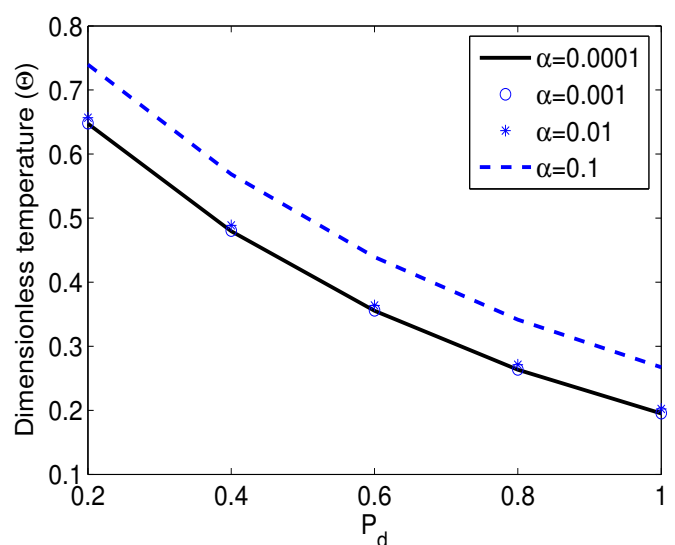

(a) At $\mathrm{x}=0, \mathrm{~F}_{0}=1.50$

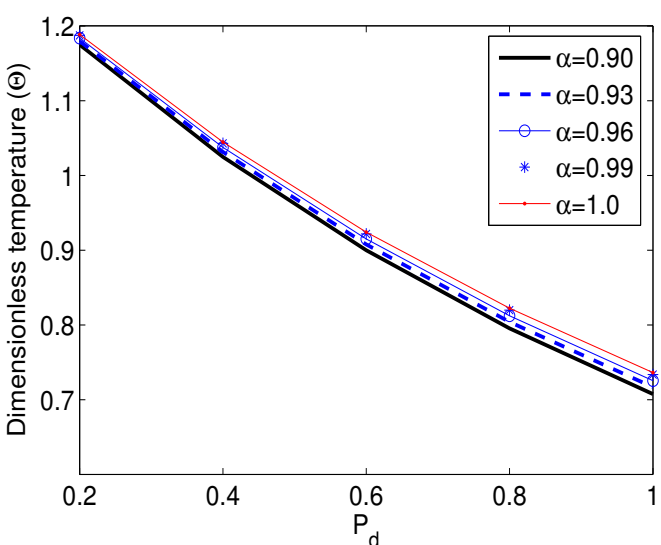

(b) At $\mathrm{x}=0, \mathrm{~F}_{0}=1.50$

Fig. 8: Variation of dimensionless temperature $(\theta)$ with $P_{d}$ 


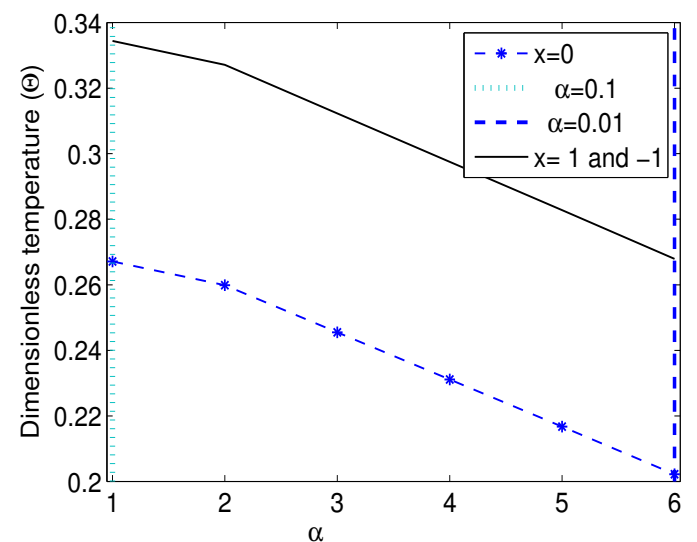

(a) At $P_{d}=1.0, F_{0}=1.50$

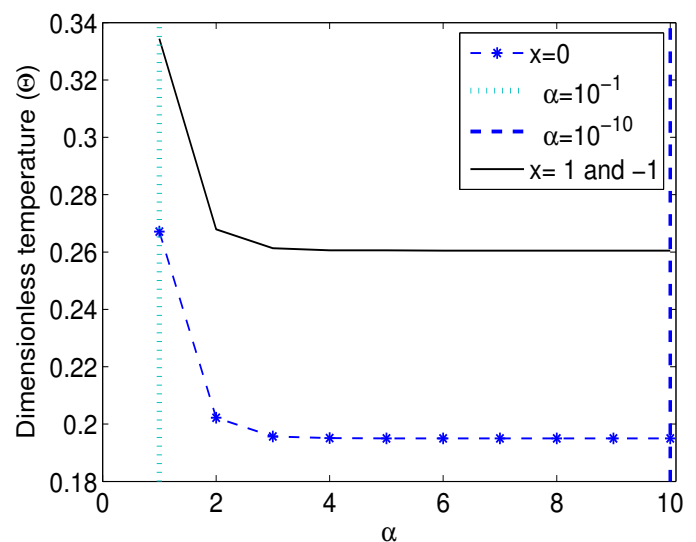

(b) At $P_{d}=1.0, F_{0}=1.50$

Fig. 9: Variation of dimensionless temperature $(\theta)$ with $\alpha$

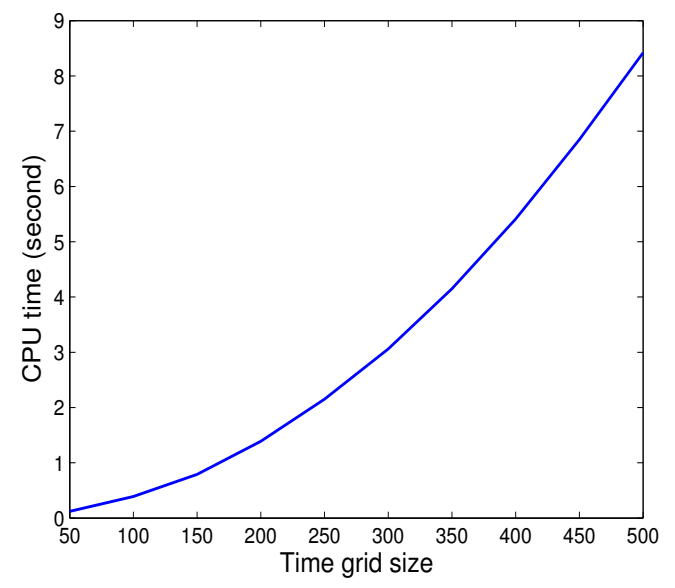

Fig. 10: Variation of CPU time with the time grid size

Fig. 8 reveals that the dimensionless temperature increases for very small increase of $\alpha$ at $F_{0}=1.50$, which shows that there is a state of $F_{0}$ and $\alpha$ at which dimensionless temperature decreases then further increases with increase of $P_{d}$, as shown in Fig. 7(b).

For very small $\alpha$ in the range of $\left(10^{-1}, 10^{-2}\right)$ dimensionless temperature decreases as shown in Fig. 9(a) and for $\alpha$ in the range $\left(10^{-1}, 10^{-10}\right)$ dimensionless temperature decreases and then takes a fixed value for any fixed $P_{d}$ and any fixed $F_{0}$, which indicate the stability of temperature as shown in Fig. 9(b).

Fig. 10 shows the executing time of our method in $2.3 \mathrm{GH}$ i3 processor with $2 \mathrm{~GB}$ ram and Windows 7 operating system.

\section{Conclusions}

A mathematical model describing time fractional heat transfer (in the sense of Caputo), in an infinite plate of finite thickness whose faces are subjected to non-homogeneous boundary condition of second kind has been analyzed using a fully implicit unconditionally stable finite difference scheme. This implicit finite difference scheme is unconditionally stable and convergent.

Our simulation shows that for $F_{0} \leq F_{0}^{*}, \theta$ decreases as $\alpha$ increases. Further, after $F_{0}>F_{0}^{*}, \theta$ increases as $\alpha \longrightarrow \alpha^{*}$, and for $\alpha>\alpha^{*}, \theta$ decreases as $\alpha$ increases. This is because of Fourier number is a measure of rate of heat conduction in comparison with the heat storage in the given volume element. Larger the Fourier number, deeper is the penetration of heat into a body over a given period of time. The Predoditelev number is defined as, $P_{d}=\frac{b R^{2}}{a\left(T_{c}-T_{0}\right)}$, due to which, $F_{0}^{*}$ increases as $P_{d}$ decreases. For any fixed $F_{0}$ and $P_{d}$, when time fractional order 
$\alpha \longrightarrow 0$, corresponding temperature tends to a fixed value showing the stability of scheme. This technique can also be applied to solve the time fractional heat conduction equation under most generalized boundary conditions.

\section{Acknowledgement}

The authors are acknowledge to the Prof. Umesh Singh, Coordinator DST-Center for Interdisciplinary Mathematical Sciences (CIMS), BHU, for providing necessary facilities.

\section{References}

[1] D. Benson, S. Wheatcraft, M. Meerschaert, Application of fractional advection-dispersion equation, Water Resour. Res., 36 (6) (2000) 1403-1412.

[2] C. Chen, F. Liu, K. Burrage, Finite difference methods and a Fourier analysis for the fractional reaction-sub-diffusion equation, Applied Mathematics and Computation, 198 (2) (2008) 754-769.

[3] C. Chen, F. Liu, I. Turner, V. Anh, A Fourier method for the fractional diffusion equation describing sub-diffusion, J. Comput. Phys., 227 (2) (2007) 886-897.

[4] H. Ding, Y. Zhang, Notes on implicit finite difference approximation for time fractional diffusion equations, Int. J. Compu. Math. Appl., 61 (2011) 2924-2928.

[5] P. Grassberger, W. Nadler, L. Yang, Heat conduction and entropy production in a one-dimensional hard-particle gas, Physical Rev. Lett., 89 (18) (2002) 180601-1-180601-4.

[6] F. Huang, F. Liu, The time fractional diffusion and advection dispersion equation, ANZIAM J. 46 (2005) $317-330$.

[7] X. Jiang, M. Xu, The time fractional heat conduction equation in the general orthogonal curvilinear coordinate and the cylindrical coordinate systems, Physica A, 389 (2010) 3368-3374.

[8] F. Khanna, D. Matrasulov, Non-linear Dynamics and Fundamental Interactions, Nato Public Diplomacy Division, 2004.

[9] T. Langlands, B. Henry, The accuracy and stability of an implicit solution method for the fractional diffusion equation, J. Comput. Phys. 205 (2005) 719-736.

[10] S. Lepri, R. Livi, A. Politi, On the anomalous thermal conductivity of one-dimensional lattices, Europhys. Lett., 43 (3) (1998) 271-276.

[11] F. Mainardi, M. Raberto, R. Goreno, E. Scalas, Fractional calculus and continuous-time finance II: the waiting-time distribution, Physica A, 287 (2000) 468-481.

[12] M. Meerschaert, D. Benson, B. Baeumer, Operator levy motion and multiscaling anomalous diffusion, Physical Rev. E, 63 (2001) 11121-11126.

[13] M. Meerschaert, D. Benson, H. Scheer, B.Baeumer, Stochastic solution of space-time fractional diffusion equations, Physical Rev. E 65 (2002) 11031-11034.

[14] D. Murio, Implicit finite difference approximation for time fractional diffusion equations, Int. J. of Compu. Math. Appl., 56 (2008) 1138-1145.

[15] M. Naber, Distributed order fractional sub-diffusion, Fractals, 12 (23) (2004) 23-32.

[16] F. Norwood, Transient thermal waves in the general theory of heat conduction with finite wave speeds, J. Appl. Mech., 39 (3) (1972) 673-676.

[17] M. Ozisik, Heat Conduction, John Wiley \& Sons, Inc, New York, 1993.

[18] I. Podlubny, Fractional Differential Equations, Academic Press, San Diego, 1999.

[19] Y. Povstenko, Fractional heat conduction equation and associated thermal stresses in an infinite solid with spherical cavity, Quarterly Journal of Mechanics and Applied Mathematics, 61 (4) (2008) 523-547.

[20] Y. Povstenko, Signaling problem for time fractional diffusion-wave equation in a half-space in the case of angular symmetry, Nonlinear Dyn., 59 (2010) 593-605. 
[21] M. Raberto, E. Scalas, F. Mainardi, Waiting-times and returns in high frequency financial data: an empirical study, Physica A, 314 (2002) 749-755.

[22] Z. Rieder, J. Lebowitz, E. Lieb, Properties of harmonic crystal in stationary nonequilibrium state, J. Mathematical Phys., 8 (1967) 1073-1078.

[23] J. Singh, P. Gupta, K. Rai, Solution of fractional bioheat equations by finite difference method and HPM, Mathematical and Computer Modelling, 54 (2011) 2316-2325.

[24] T. Solomon, E.Weeks, H. Swinney, Observation of anomalous diffusion and levy flights in a 2-dimensional rotating flow, Physical Rev. Lett., 71 (24) (1993) 3975-3978.

[25] S. Yuste, Weighted average finite difference methods for fractional diffusion equations, J. Comput. Phys., 216 (2006) 264-274.

[26] S. Yuste, L. Acedo, An explicit finite difference method and a new von Neumann-type stability analysis for fractional diffusion equations, SIAM J. Numer. Anal., 42 (5) (2005) 1862-1874.

[27] S. Yuste, K. Lindenberg, Sub-diffusion-limited A+A reactions, Physical Rev. Lett., 87 (2001) 118301-118304.

[28] Y. Zhang, A finite difference method for fractional partial differential equation, Applied Mathematics and Computation, 215 (2009) 524-529.

[29] X. Zhao, Z. Sun, A box-type scheme for the fractional sub-diffusion equation with Neumann boundary conditions, J. Comput. Phys., 230 (2011) 6061-6074.

[30] P. Zhuang, F. Liu, Implicit difference approximation for the time fractional diffusion equation, Journal of Applied Mathematics and Computing, 22 (3) (2006) 87-99.

[31] P. Zhuanga, F. Liu, V. Anh, I. Turner, New solution and analytical techniques of the implicit numerical method for the anomalous sub-diffusion equation, SIAM J. Numer. Anal., 46 (2) (2008) 1079-1095. 